JOURNAL DE PHYSIQUE IV

Colloque C4, supplément au Journal de Physique III, Vol. 1, novembre 1991

\title{
STRESS-INDUCED PHASE TRANSFORMATIONS IN RATE-TYPE VISCOELASTICITY
}

\author{
C. FACIU(1) \\ Institute of Mathematics, str. Academiei 14, 70109 Bucharest, Romania
}

\begin{abstract}
A Maxwell's rate-type viscoelastic constitutive equation is used to describe stress-induced phase transitions of shape memory alloys in an one-dimensional body. A simplified piecewise linear load-deformation equilibrium curve of "van der Waals type" is prescribed and the non-convex free-energy of the model is determined. We consider two classical types of loading devices: a hard device and a soft device.Energetic estimates and numerical solutions for the corresponding problems are given.
\end{abstract}

\section{1.-Introduction and problem description}

Studies of the pseudo-elastic hysteresis, that occurs in the austenitic-martensitic phase transformations of shape memory alloys have indicated that the main features of these transitions are predicted by the use of a non convex stored energy, or equivalently, a non-monotone stress-deformation relation. See for example the simple Landau-Devonshire model which Falk /3/ has applied to shape memory alloys and also the statistical-mechanical theory developped by Müller $/ 2 /$ for a pseudo-elastic body that provides non-convex free energy curves. On the other hand, in the last years the energetic study of rate-type viscoelastic constitutive equations seems to lead to a better description of the phase transitions phenomena by means of such constitutive equations with a non-monotone equilibrium curve (see for instance Suliciu /3/, Faciu /4/ and the references given there).

In this paper we shall present some energetic estimates and numerical solutions for the initial-boundary value problems which correspond to the classical types of loading devices in the dynamic rate-type viscoelastic theory of bars: the soft device and the hard device. Comparaison with physical experiments reported in the literature shows that the model may describe qualitatively well different characteristics of a superelastic loop such as the presence or absence of a peak at the yield point and a sawtooth-like deformation on the horizontal branches of the loops. The model is also able to describe the inhomogeneities which occur in the uniaxial hard-tension tests and the propagation of phase boundaries.

We consider one of the simplest geometrical situation of the dynamic theory of bars when the motion of an onedimensional body is described by the balance of momentum and by the compatibility equation

$$
\rho \frac{\partial \mathrm{v}}{\partial \mathrm{t}}-\frac{\partial \sigma}{\partial \mathrm{x}}=0, \quad \text { and } \quad \frac{\partial \varepsilon}{\partial \mathrm{t}}-\frac{\partial \mathrm{v}}{\partial \mathrm{x}}=0
$$

where the unknown functions $v, \sigma$ and $\varepsilon$ are the particle velocity, the stress and the strain, respectively. $\rho=$ const. $>0$ is the mass density of the material in a spatially homogeneous reference configuration, $x$ is the spatial Lagrangian coordinate and $t \in R^{+}$is the time. The system (1) must be completed by a constitutitve equation relating the stress $\sigma$ and the strain $\varepsilon$. It is well known that the classical phenomenological description of transitions between the liquid and vapor phases was generally made, by using the constitutive model of a thermo-elastic fluid of van der Waals type. By analogy, the phenomenon of phase transformations in solid bars (for example, austenitic-martensitic phase transition of shape memory alloys) was modeled by certain authors (see for example $/ 5 /$ ) by means of a non-monotone elastic constitutive equation

$$
\sigma=\sigma_{\mathrm{R}}(\varepsilon)
$$

Thus, function $\sigma_{R}()$ is defined on an interval $I, 0 \in I, \sigma_{R}(0)=0, \sigma_{R}(\cdot) \in C^{2}(I)$ and for the assigned constants $\varepsilon_{\alpha}$ and $\varepsilon_{\beta}$ consistent with the requirement $0<\varepsilon_{\alpha}<\varepsilon_{\beta}$ one supposes that

$$
\text { i) } \sigma_{R}{ }^{\prime}(\varepsilon)>0 \text { on }\left[0, \varepsilon_{\alpha}\right) \cup\left(\varepsilon_{\beta}, \infty\right) \quad \text { and } \quad \text { ii) } \sigma_{R}{ }^{\prime}(\varepsilon)<0 \text { on }\left(\varepsilon_{\alpha}, \varepsilon_{\beta}\right)
$$

(1) Present address: Physique et Mécanique des Matériaux, 101, rue de la Physique, Domaine Universitaire, BP. 46, F-38402 St Martin d'Hères cedex, France 
Usually, the constitutive function restricted to the interval $\left[0, \varepsilon_{\alpha}\right)$ is called the $\alpha$-branch and the restriction of the constitutive function to the interval $\left(\varepsilon_{\beta}, \infty\right)$ is called the $\beta$-branch. If at some pair $(x, t)$ the value of $\varepsilon(x, t)$ lies in the interval $\left(0, \varepsilon_{\alpha}\right)$ (or in the interval $\left(\varepsilon_{\beta}, \infty\right)$, then one says that the point $x$ is in the $\alpha$-phase ( $\beta$-phase) at time $t$. If the value of $\varepsilon(x, t)$ lies in the interval $\left(\varepsilon_{\alpha}, \varepsilon_{\beta}\right)$ then one says that the point $\mathrm{x}$ is in the spinodal region $\left(\varepsilon_{\alpha}, \varepsilon_{\beta}\right)$.

From mathematical point of view, the above elastic system $(1+2)$, which governs the motion of the body has the disadvantage that it leads to a mixed hyperbolic-elliptic initial value problem. The most obvious difficulty is then related to the Hadamard's argument of ill-possedness of the Cauchy problem in the elliptic regime. From thermodynamical point of view the most important disadvantage of the elastic model seems to be that the system is not able to describe how the stress may deviate from the equilibrium.One way to circumvent all these inconveniences is to use a Maxwell's type viscoelastic model (see Suliciu / $3 /$ and Faciu /4/) of the form

$$
\dot{\sigma}=\mathrm{E} \dot{\varepsilon}+\mathrm{G}(\varepsilon, \sigma)
$$

which relates the stress history $\sigma=\sigma(\mathrm{x}, \mathrm{t})$ to the strain history $\varepsilon=\varepsilon(\mathrm{x}, \mathrm{t})$ of a given particle $\mathrm{x}$ of the body and where the dot signifies the time derivative. $\mathrm{E}=$ const. $>0$ is the dynamic Young's modulus and characterizes the instantaneous response of the material and the relaxation modulus $\mathrm{G}$ may be taken in the simplest form

$$
\mathrm{G}(\varepsilon, \sigma)=-\mathrm{k}\left(\sigma-\sigma_{\mathrm{R}}(\varepsilon)\right) \text {, }
$$

i.e. linear dependent on the overstress function. Here $k=$ const. $>0$ is a Maxwell's type viscosity coefficient and $1 / k$ is a relaxation time. The equilibrium load-deformation curve of the viscoelastic model is supposed to be a non-monotone one , with the same properties i) and ii) as the elastic curve (2). This model has the capacity to describe qualitatively general facts that are experimentally observed in shape memory alloys like creep,relaxation phenomena, inverse creep which cannot be described by means of the constitutive equations of elasticity and classical plasticity. Moreover, the viscoelastic system $(1+3)$ has the advantage that it is always hyperbolic irrespective of the shape of the equlibrium curve $\sigma=\sigma_{R}(\varepsilon)$, since $E>0$. Thus the initial-boundary value problems are theoretically well-posed and the Hadamard's observation that the wave speed ceases to be real if the slope of the curve $\sigma=\sigma_{R}(\varepsilon)$ become negative is overcome. However, as it was shown in a paper by Suliciu /3/ and as can be seen from the numerical experiments performed by Faciu $/ 4 /$ in the case of the familiar van der Waals equation of state, the system $(1+3)$ incorporates some physical instabilities due to the shape of the equilibrium curve. These instabilities allow the model to describe differents characteristics of a superelastic loop which will be discussed in the next sections. Another important point is that the viscoelastic model $(3+4)$ can describe the way the state $(\varepsilon, \sigma)(x, t)$ may deviate from the equilibrium states $\sigma(x, t)=\sigma_{R}(\varepsilon(x, t))$, while the elastic one does not.

\section{2.-The free energy function}

In this section we introduce the notion of free energy function and some of its properties. We construct this energy function for the simplified viscoelastic piecewise linear model in order to illustrate how changes the free energy function when the equilibrium curve modifies its shape.

The constitutive equation (1), is said to possess a free energy of strain and stress, compatible with the second law of themodynamics, if there exists a smooth function $\psi=\psi(\varepsilon, \sigma)$ such that $\rho \psi(\varepsilon, \sigma) \leq \sigma \varepsilon$, for any process $(\varepsilon(t), \sigma(t)), t \in[0, T)$. One can prove (see $/ 6 /$ ) that there is a unique free energy function $\psi=\psi(\varepsilon, \sigma)$, verifying $\psi(0,0)=0$, and

$$
\frac{\partial \psi}{\partial \varepsilon}+E \frac{\partial \psi}{\partial \sigma}=\frac{\sigma}{\rho} ; \quad \frac{\partial \psi}{\partial \sigma}(\varepsilon, \sigma) G(\varepsilon, \sigma) \leq 0
$$

if and only if the slope of the straightline connecting any two points on the equilibrium curve is bounded from above by the instantaneous Young's modulus. Moreover, one shows that

$$
\rho \psi(\varepsilon, \sigma)=\sigma^{2} / 2 E+\varphi(\sigma-E \varepsilon)
$$

is a solution of (5) where $\varphi\left(\right.$ ) is an arbitrary smooth function of its argument. Since $\frac{\partial \psi}{\partial \sigma}\left(\varepsilon, \sigma_{\mathrm{R}}(\varepsilon)\right)=0$, for any $\varepsilon \in \mathrm{I}$, we deduce that

$$
\varphi^{\prime}\left(\sigma_{\mathrm{R}}(\varepsilon)-\mathrm{E} \varepsilon\right)=-\sigma_{\mathrm{R}}(\varepsilon) / \mathrm{E}
$$

Let us consider the simpliefied case of a continuous piecewise linear function $\sigma=\sigma_{R}(\varepsilon)$ defined as follows:

$$
\sigma_{R}(\varepsilon)= \begin{cases}E_{1} \varepsilon, & \text { for } 0<\varepsilon<\varepsilon_{\alpha} \\ -E_{2}\left(\varepsilon-\varepsilon_{\alpha}\right)+\sigma_{\alpha} & \text { for } \varepsilon \in\left(\varepsilon_{\alpha} \varepsilon_{\beta}\right) \\ E_{3}\left(\varepsilon-\varepsilon_{\beta}\right)+\sigma_{\beta}, & \text { for } \varepsilon>\varepsilon_{\beta}\end{cases}
$$

The entries of $\sigma_{\mathrm{R}}(\varepsilon)$ and $\mathrm{E}$, which may depend on the absolute temperature, are required to satisfy the restrictions $0<E_{1}<E, 0<E_{3}<E$, where $0<\varepsilon_{\alpha}<\varepsilon_{\beta}, \sigma_{\alpha}>0, \sigma_{\alpha}-\sigma_{\beta}=-E_{2}\left(\varepsilon_{\alpha}-\varepsilon_{\beta}\right), \sigma_{\alpha}=E_{1} \varepsilon_{\alpha}$.

We can show that function $\varphi(\cdot)$ is given in this case by relation 


$$
\varphi(\tau)=\left\{\begin{array}{lr}
\tau^{2} \mathrm{E}_{1} / 2 \mathrm{E}\left(\mathrm{E}-\mathrm{E}_{1}\right), & \tau_{\alpha} \leq \tau \leq 0 \\
-\tau^{2} \mathrm{E}_{2} / 2 \mathrm{E}\left(\mathrm{E}+\mathrm{E}_{2}\right)-\tau\left(\mathrm{E}_{2} \varepsilon_{\beta}+\sigma_{\beta}\right) /\left(\mathrm{E}+\mathrm{E}_{2}\right)+\varphi_{1}, & \tau_{\beta} \leq \tau<\tau_{\alpha} \\
\tau^{2} \mathrm{E}_{3} / 2 \mathrm{E}\left(\mathrm{E}-\mathrm{E}_{3}\right)-\tau\left(-\mathrm{E}_{3} \varepsilon_{\beta}+\sigma_{\beta}\right) /\left(\mathrm{E}-\mathrm{E}_{3}\right)+\varphi_{2}, & \tau<\tau_{\beta}
\end{array}\right.
$$

where $\varphi_{1}=-\tau_{\alpha}^{2}\left(E_{1}+E_{2}\right) / 2\left(E+E_{2}\right)\left(E-E_{1}\right), \varphi_{2}=\varphi_{1}+\tau_{\beta}^{2}\left(E_{3}+E_{2}\right) / 2\left(E+E_{2}\right)\left(E-E_{3}\right), \tau_{\alpha}=\left(E_{1}-E\right) \varepsilon_{\alpha}>\tau_{\beta}=\sigma_{\beta}-E \varepsilon_{\beta}$

Therefore we have the following result, established in $/ 6 /$ in a more general case. The function $\psi(\varepsilon, \sigma)$ given by $(6)$ with $\varphi$ determined by $(7)$ is the unique function verifying $(5)_{2}$ with $\psi(0,0)=0$. Moreover, if we denote the free energy at equilibrium by $\psi_{R}(\varepsilon)=\psi\left(\varepsilon, \sigma_{R}(\varepsilon)\right)$ we see from (6) and (7) that $\frac{d \psi_{R}}{d \varepsilon}(\varepsilon)=\frac{1}{\rho} \sigma_{R}(\varepsilon)$, i.e., the equilibrium free energy of the viscoelastic model is exactly the free energy of the elastic model. The free energy function $\rho \psi(\varepsilon, \sigma)$ of the viscoelastic model is composed by a quadratic term in stress and by function $\varphi$. We can observe that function $\varphi$ has the same properties as the free energy for the simple Landau-Devonshire model which Falk /1/ has applied to shape memory alloys. Therefore, we expect that our non-monotone load-deformation equilibrium curve may describe pseudo-elastic loops that occur in the austenitic-martensitic phase transition. Indeed, from relation ( 9 ) and the construction of the free energy function it is easy to see that the states $S=\left(\varepsilon, \sigma_{R}(\varepsilon)\right)$, where the equilibrium curve $\sigma=\sigma_{R}(\varepsilon)$ changes the monotony correspond to the inflexion points $S=\left(\tau=\varepsilon-E \sigma_{R}(\varepsilon), \varphi(\tau)\right)$ of the function $\varphi$; the states $S=\left(\varepsilon, \sigma_{R}(\varepsilon)\right)$ where the equilibrium curve $\sigma=\sigma_{R}(\varepsilon)$ changes its sign correspond to the extremum points $s=\left(\tau=\varepsilon-E \sigma_{R}(\varepsilon), \varphi(\tau)\right)$ of function $\varphi$ (see for example Fig.1).
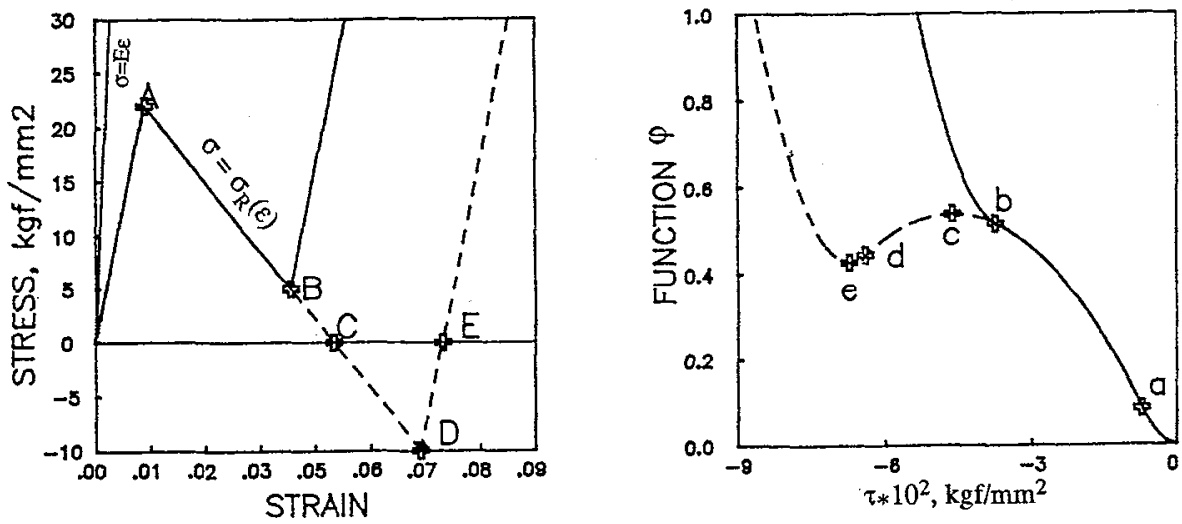

Fig.1 The change in free-energy (eq. (6)) that results from a change in the equilibrium curve.

\section{3. - Energy estimates and numerical solutions}

One can prove (see $/ 6 /$ and the references given there) that the following energy identity

$$
\rho \frac{\partial \mathrm{e}^{*}}{\partial \mathrm{t}}-\frac{\partial(\sigma \mathrm{v})}{\partial \mathrm{x}}-\rho \frac{\partial \psi}{\partial \sigma}(\varepsilon, \sigma) \mathrm{G}(\varepsilon, \sigma)=0
$$

holds for all smooth solutions of the system $(1+3)$. Here $e^{*}=e^{*}(\sigma, \varepsilon, v)=v^{2} / 2+\psi(\varepsilon, \sigma)$ is the density of the total energy of the system $(1+3)$. The energy identity (10) will allow us to draw important conclusions about the behaviour of the solutions of the viscoelastic system if we know when $\psi(\varepsilon, \sigma)$ is positive at all states $(\varepsilon, \sigma)$

It will be of interest to see what will predict our rate-type viscoelastic model $(3+4)$ when we simulate the followings two classical tension tests.

a) Hard device.

We consider first that a bar of lenght $l$ is firmly fixed at one end, the other being moved with a speed $v_{\mathrm{I}}(t)$. This means that we have to determine the solution of the following initial-boundary value problem

$$
\varepsilon(x, 0)=\varepsilon_{0}(x), \sigma(x, 0)=\sigma_{0}(x), v(x, 0)=v_{0}(x), \text { for } x \in[0,1] \text { and } v(0, t)=v_{1}(t), v(1, t)=0, \quad \text { for } t \in[0, T)
$$

for the system $(1+3)$. When the speed $v_{1}(t)=v^{*}=$ const. this problem corresponds to the usual physical experiment performed in laboratory when a bar is deformed at the constant average strain rate $-v^{*} / 1$.

By making the following change of dependent variables $V(x, t)=v(x, t)-v_{1}(t)(1-x / l)$ and using relations $(10)$ and $(5)_{2}$ we can show that the solution of the problem (11) satisfies the following inequalities (see/3/ and/4/) 


$$
\sqrt{e(t)} \leq \sqrt{e(0)}+\sqrt{2} / 2 \int_{0}^{t}\left(\frac{E}{1} v_{1}^{2}(s)+\frac{1}{3} \dot{v}_{1}^{2}(s)\right) d s
$$

and

$$
\int_{0}^{t} \int_{0}^{1}\left(\sigma-\sigma_{R}(\varepsilon)\right)^{2} d x d s \leq \frac{E+E_{2}}{k}\left\{\left[\sqrt{e(0)}+\sqrt{2} / 2 \int_{0}^{t}\left(\frac{E}{1} v_{1}^{2}(s)+\frac{1}{3} \dot{v}_{l}^{2}(s)\right) d s\right]^{2}-e(t)\right\}
$$

where $e(t)=\int_{0}^{1}\left(V^{2} / 2+\psi(\varepsilon, \sigma)\right)(x, t) d x, t \geq 0$ is the total energy of the problem (11)

The energetic inequality (12) shows that, for any given function $v_{1}(t), t \geq 0$ such that $v_{1}^{2}(s)$ and $\dot{v}_{1}^{2}(s)$ are integrable on any finite time interval, the total energy $\mathrm{e}(\mathrm{t})$ is bounded by an energetic measure of the input data and inequality (13) says that there is an approach to the equilibrium in $L^{2}$ sense when $k \rightarrow \infty$, i.e., when we want to consider the elastic problem as a limiting case of the viscoelastic problem.

We shall be interested to study the exact solution of the problem (11) in the special case when

(11) $\quad \varepsilon_{0}(x)=0, \sigma_{0}(x)=0, v_{0}(x)=v^{*}(1-x / l)$, for $x \in[0,1]$ and $v(0, t)=v^{*}=$ const. $<0, v(1, t)=0$, for any $t \in[0, T)$ i.e., when the bar is in the natural state $(\varepsilon=0, \sigma=0)$ at the initial moment and the left end is moved with the constant speed $v^{*}$.

In paper by Suliciu $/ 3 /$, (see also Faciu /4/) it was shown that, irrespective of the slope of the equilibrium curve, there exists spatially homogeneous solutions in stress and strain of the form

$$
v(x, t)=v^{*}(1-x / l), \quad \varepsilon(x, t)=-\frac{v^{*}}{1} t+\varepsilon_{0}, \quad \sigma(x, t)=\sigma(t), \quad \text { for any } x \in[0,1] \text { and } t \in[0, T)
$$

for the initial-boundary data problem $(11)^{\prime}$, where $\sigma=\sigma(t)$ is determined as the solution of the differential equation

$$
\dot{\sigma}(\mathrm{t})=-\mathrm{Ev} * / 1-\mathrm{k}\left(\sigma(\mathrm{t})-\sigma_{\mathrm{R}}(\varepsilon(\mathrm{t})), \quad \sigma(0)=\sigma_{\mathrm{o}}\right.
$$

Then, in order to study the homogeneous solution (14-15) it suffices to study the Cauchy problem :

$$
\frac{\mathrm{d} \hat{\sigma}(\varepsilon)}{\mathrm{d} \varepsilon}=-\mathrm{E}+\frac{\mathrm{kl}}{\mathrm{v}^{*}}\left(\hat{\sigma}(\varepsilon)-\sigma_{\mathrm{R}}(\varepsilon)\right), \quad \hat{\sigma}\left(\varepsilon_{\mathrm{o}}\right)=\sigma_{\mathrm{o}}
$$

where $\hat{\sigma}(\varepsilon)=\sigma\left(\left(\varepsilon_{\mathrm{o}}-\varepsilon\right) \mathrm{l} / \mathrm{v}^{*}\right)$.

If we study the behaviour of $\hat{\sigma}(\varepsilon)$ with respect to the equilibrium curve $\sigma=\sigma_{\mathrm{R}}(\varepsilon)$ (see for example $/ 3 /$ and $/ 4 /$ ) we can establish an estimate of the following type

$$
\begin{aligned}
0 & \leq\left(\hat{\sigma}(\varepsilon)-\sigma_{\mathrm{R}}(\varepsilon)\right) \leq \frac{E v^{*}}{\mathrm{kl}}, \text { for } \varepsilon \in\left[0, \varepsilon_{\alpha}\right) \text { or } \varepsilon \in\left[\varepsilon_{\alpha,} \infty\right) \\
\frac{E v^{*}}{\mathrm{k} l} & \leq\left(\hat{\sigma}(\varepsilon)-\sigma_{\mathrm{R}}(\varepsilon)\right) \leq \frac{\left(\mathrm{E}+\mathrm{E}_{2}\right) \mathrm{v}^{*}}{\mathrm{kl}}, \text { for } \varepsilon \in\left(\varepsilon_{\alpha}, \varepsilon_{\beta}\right)
\end{aligned}
$$

Therefore a continuous transition in time from phase $\alpha$ to phase $\beta$ (or vice versa) spatially homogeneous in $\varepsilon$ and $\sigma$ can be realised along a path which follows the equilibrium curve however close we want. Indeed, according to the estimates (17) the homogeneous solution will be closer to the equilibrium curve $\sigma=\sigma_{R}(\varepsilon)$ for larger viscosity coefficient $k$ and/or slower piston velocity $\mathrm{v}^{*}$. problem.

From experimental point of view, it will be of interest to consider the more realistic initial-boundary value (18) $\quad \varepsilon_{0}(x)=0, \sigma_{0}(x)=0, v_{0}(x)=0$, for $x \in[0,1]$ and $v(0, t)=v^{*}=$ const. $<0, v(1, t)=0, \quad$ for any $t \in[0, T)$.i.e., we consider the bar initially at rest, then suddenly apply a constant particle velocity at $x=0$ and hold fixed the end $x=1$. Since the viscoelastic system $(1+3)$ is always byperbolic the method of characteristics is very useful to obtain numerical solutions for the initial-boundary value problems for this system. When the time integration step was chosen to verify the restrictions implied by the second law of thermodynamics, as described in $/ 7 /$ and the input data were exactly (18), then the numerically found solution was exactly the homogeneous solution described above(see also /4/). But, when some small perturbation was introduced into the input data, for example by the initial shock introduced at the initial moment in (18), then the spatial homogeneity of stress and strain was lost in the interval $\left(\varepsilon_{\alpha}, \varepsilon_{\beta}\right)$ and a wavy phenomena started to build up.

In the numerical calculations performed in this paper we have used the following entries: $E=9500 \mathrm{kgf} / \mathrm{mm} 2$, $E_{1}=E_{2}=2200 \mathrm{kgf} / \mathrm{mm}^{2}, E_{2}=566.6 \mathrm{kgf} / \mathrm{mm}^{2}, \rho=7.5 * 10^{-} 6 \mathrm{~kg} / \mathrm{mm}^{3}, 1=20 \mathrm{~mm}, v^{*}=-10 \mathrm{~mm} / \mathrm{sec}$. Therefore the average strain rate was $\varepsilon_{\mathrm{m}}=0.5 / \mathrm{sec}$. 
From the numerical experiments presented here (see also $/ 4 /$ ) we observe that always when a small perturbation is present we may conduct a spatially homogeneous process only on the ascending branches of the equilibrium curve (i.e., when $\sigma_{R}^{\prime}(\varepsilon)>0$ ). In the interval $\left(\varepsilon_{\alpha}, \varepsilon_{\beta}\right)$ this homogeneity is very sensitive to any small perturbation and it is easily lost, but it is recovered again when this interval is leaved. See for example Fig 2. where a sawtooth-like deformation is observed. When the loading test, at the constant average strain rate $-v^{*} /$, illustrated in this figure is followed by an unloading test at the oposite average strain rate $v^{*} /$, then we may put into evidence a hysteretic behaviour of the solution. This behaviour agrees qualitatively with the macroscopic and microscopic observations of stress-induced martensitic transformations reported by Otsuka and all. /8/ in a specimen of $\mathrm{Cu}-\mathrm{Al}-\mathrm{Ni}$ single crystal during a tensile test.

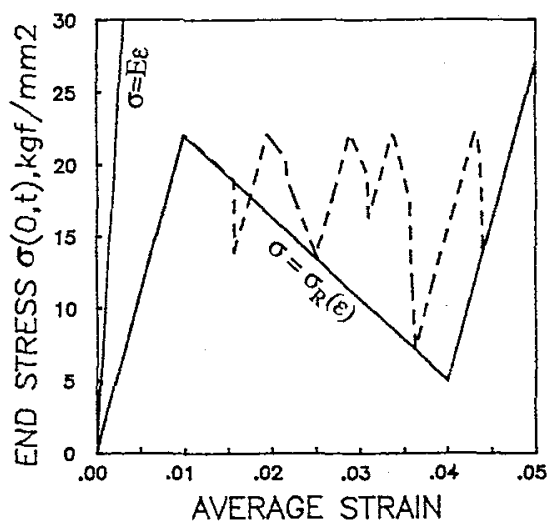

Fig. 2

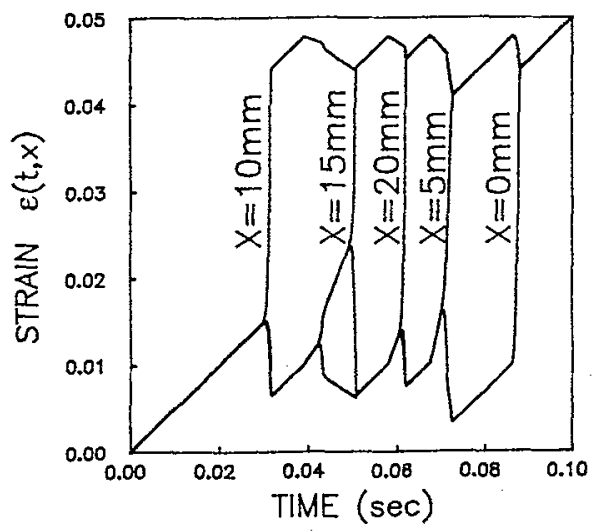

Fig. 3

Therefore, our rate-type mode $(4+5)$ is able to describe different characteristics of the super-elastic loop in a hard testing machine experiment like the presence of a sharp peak at the yield point, the fluctuations observed in /8/ on the horizontal branches of the loop and also the strain localization.

According to Otsuka and all. /8/ martensite formation is inhomogeneous and ressembled to Lüders band propagation. Our numerical experiments agree very well with these facts, and we can describe the successive formation and propagation of strain bands through the specimen. In order to illustrate the way the strain localized successively, in Fig. 3 we have depicted the strain history for different points of the bar.

The way the phase boundaries move through the specimen in a uniaxial tension test converting regions of moderate stretch into regions of high stretch will be analysed elsewhere.

It is also of interest to remark that when a point $x$ of the bar $x \in(0,1)$ passes through the spinodal region it supports high accelerations (see Fig.4). Therefore, even if the strain rate of the process is small it is unreasonable to neglige the inertial terms in equations (1).

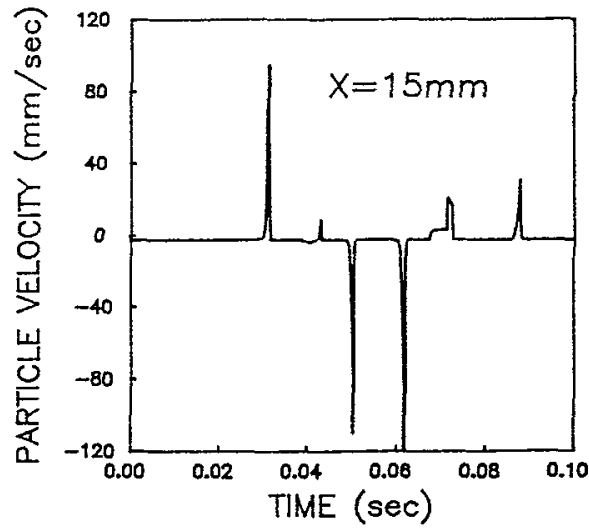

Fig. 4 
b) Soft device

We are interested now to simulate a soft-testing machine experiment. In this case the bar is firmly fixed at one end, the other being subjected to a given slightly increasing and then decreasing load $\sigma_{1}(t)$, i.e. we have to determine the solution of the following initial-boundary value problem

$$
\begin{aligned}
& \varepsilon(x, 0)=0, \sigma(x, 0)=0, v(x, 0)=0, \text { for any } x \in[0,1], \\
& \sigma(0, t)=\sigma_{1}(t)=\left\{\begin{array}{ll}
a t, & \text { for } t \in\left[0, t_{o}\right), \\
-a\left(t-t_{0}\right)+2 t_{o}, & \text { for } t \in\left(t_{o}, 2 t_{o}\right)
\end{array} \quad \text { for } t \in\left[0,2 t_{0}\right)\right.
\end{aligned}
$$

where $\mathrm{a}=1000 \mathrm{kgf} / \mathrm{mm}^{2} \mathrm{sec}$ and $\mathrm{t}_{\mathrm{o}}=0.025 \mathrm{sec}$.

In this case, using again the properties of the free energy function (6) and relation $(5)_{2}$ we deduce the following energy inequality verified by the smooth solutions of the soft problem (19) for the viscoelastic system $(1+3)$

$$
\frac{1}{1} \mathrm{e}(\mathrm{t}) \leq \frac{1}{1} \mathrm{e}(0)+\int_{0}^{\mathrm{t}} \sigma_{1}(\mathrm{~s}) \dot{\varepsilon}_{\mathrm{m}}(\mathrm{s}) \mathrm{ds}
$$

where $e(t)=\int_{0}^{1}\left(v^{2} / 2+\psi(\varepsilon, \sigma)\right)(x, t) d x, \geq 0$ is the total energy of the bar at time $t$ and $\dot{\varepsilon}_{m}(t)=\int_{0}^{1} \dot{\varepsilon}(x, t) d x$ is the average strain rate.

This energetic inequality shows that the average energy of the body at time $t$ is less than the average energy of the initial data plus the work done on the entire body by the applied load $\sigma_{1}(t)$. We must remark that if the curve $\left(\sigma_{1}(t), \varepsilon_{m}(t)\right)$, $t \in[0, T)$ is closed, i.e. $\sigma_{1}(0)=\sigma_{1}(T)$ and $\varepsilon_{\mathrm{m}}(0)=\varepsilon_{\mathrm{m}}(\mathrm{T})$, then the second term in inequality (20) is exactly the area of the hysteresys loop. See Fig. 5 which shows that a non-monotone equilibrium curve of the visocoelastic model may give rise to a superelastic loop.

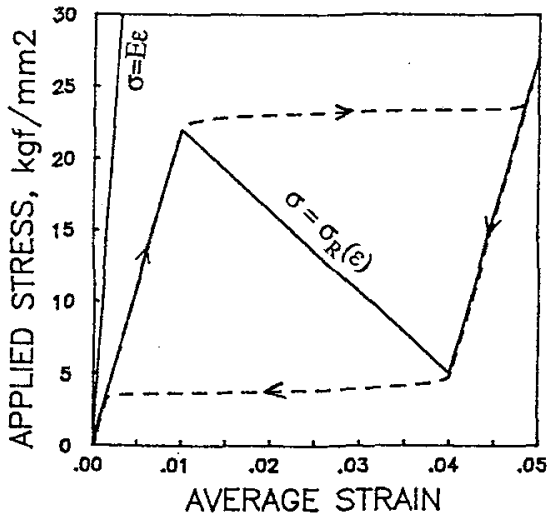

Fig. 5 Superelastic loop in the softtesting experiment (19).

Acknowledgements -I gratefully acknowledge a fellowship of the french government at Laboratoire Génie Physique et Mécanique des Matériaux, URA CNRS No. 793, I.N.P. Grenoble, where part of this work was carried out.

\section{REFERENCES}

/1/ FALK,F.,Acta Metalurgica,28, 1773-1780(1980)

2/MULLER,I., Pseudoelasticity in shape memory alloys-An extreme case of thermoelasticity, Proc. Symp. "Termoelasticita Finita", Acc. Naz. Lincei, 76, Rome(1986).

/3/Suliciu,I., Some stability-instability problems in phase transitions modelled by piecewise linear elastic or viscoelastic constitutive equations, Cont. Mech. Thermodyn.(to appear)

/4/FACIU,C., Int. J. Engng. Sci., 29,1103-1119(1991)

/5/ERICKSEN, J.L., J. Elasticity,5, 191-201(1975)

16/FACIU,C. ,MiHailescu-Suliciu,M., Int. J. Solids Structures,23,1505-1520(1987).

7/Mihallescu-Suliciu,M. Suliciu,I., ZAMM,10,479-486 (1985); On the method of characteristics in rate-type viscoelasticity. Non-monotone equilibrium case.(to appear ZAMM)

18/OTsuka,K., WAYMan,C.M., NAKAI,K., SAKamoto,H., ShIMIZU,K., Acta Metalurgica,24, 207-226(1976) 